\title{
GV Technology for Exploration and Delineation of Lode Gold Claims
}

\author{
Jim Combs ${ }^{1}$ and Jeffrey Drizin ${ }^{2}$ \\ 1. Geo Vision, Inc., 12945 Vanderbilt Drive \#207, Naples 34110, FL, USA \\ 2. Geo Vision, Inc., 1910 Browning Court, Highland Park 60035, IL,USA
}

\begin{abstract}
Demands for new gold deposits are increasing worldwide and the need to better define lode and placer claims is a critical environmental and economic issue. Recently, interesting results have been produced using dedicated geological information systems designed to explore for earth mineral deposits. These systems employ properties of fundamental physical fields generated by interactions of superlight elementary particles. GV technology (Geo-Vision geological direct detection radiometric and Air-Gamma technology), a revolutionary technology for exploring the earth from outer space and from inside aircraft, has been utilized to identify and precisely locate gold deposits. All elements of the Earth's crust exist in an excited state and are constantly emitting what can be called their "intrinsic radiation". The melding of science and data interpretation makes GV Technology ideal for defining both lode and placer gold deposits. The use of GV technology will improve success ratios associated with the exploration for and delineation of gold deposits and provide better definition of successful core drilling locations. The technology will also decrease the cost of defining gold deposits and since GV technology is ecologically friendly, it provides considerable reductions in environmental mitigation costs. From 1995 to 2014, GV technology has been used to identify and delineate gold deposits in Russia, Mongolia, Kirghizia, Tanzania, Ethiopia, Namibia, Australia, and the United States.
\end{abstract}

Key words: GV technology, intrinsic radiation, super small subatomic particles, satellite imagery, gold exploration.

\section{Introduction}

During the last decade, there has been a significant decline both in the number of major gold deposits discovered (> 2.5 Moz Au) and the amount of gold contained in these deposits, compared to discoveries in the early to mid 1990s [1]. In addition to declining discovery rates, future success will be achieved in an environment of increasing costs, increasing need for yearly replacement of reserves/resources, and an apparent minimum size of deposits that really impact the bottom line in larger companies [2].

GVI (Geo Vision Inc.), consisting of engineers, scientists, and management personnel, is engaged in expanded use of GV technology (Geo-Vision geological direct detection radiometric and Air-Gamma technology) in the exploration for earth mineral deposits. Recently, there have been interesting

Corresponding author: Jim Combs, Ph.D., research field: geophysics \& satellite imagery. E-mail: jim@gvdetection.com. results produced in the utilization of dedicated geological information systems designed to explore earth mineral deposits. The systems employ properties of fundamental physical fields generated by the weak interaction of superlight elementary particles. GV technology is a revolutionary technology for exploring the earth from outer space and from inside aircraft to identify and locate precisely gold, silver, hydrocarbons, geothermal, and many other earth mineral deposits. The technology reveals, and thereafter examines specifically energy emanation of earth minerals in any given area of the globe. The basic nature of GV technology rests on the premise that all physical objects-planetary and outer space-have unique properties of energy represented by light elementary 3SP (super small subatomic particles) from the lepton family of super-light subatomic particles. These super small particles can be tracked, or at least the reflections and echoes can be 
tracked. Gold, silver, coal, hydrocarbons, and geothermal energy sources all generate these particles and leave unique signatures, which can be observed on satellite images.

The science of GV technology springs from the fertile scientific minds at the A.F. Okhatrin Laboratory in Russia though guarded from the West for decades. The wider scientific world has yet to accept theoretical justifications for these effects, as well as Okhatrin's conceptual model. Unfortunately, scientific acceptance is a slow process. But in its defense, GV technology field applications in mineral discovery based on the original findings of Okhatrin have been so precise and successful that the underlying value of the science seems inescapable.

Okhatrin's underlying concept postulates the existence of SSP (super subatomic particles) and a product of the natural decay of a substance. It has leptonic properties, but with a significantly smaller mass. Leptons are elementary particles and a fundamental constituent of matter. The best known of all leptons is the electron, which governs nearly all of chemistry as it is found in atoms and is directly tied to all chemical properties. Two main classes of leptons exist: charged leptons (also known as the electron-like leptons), and neutral leptons (better known as neutrinos). More than 200 different subatomic particles have been found, however, scientists are still looking for additional ones. In fact, more than 400 elementary subatomic particles “exist” (accelerons, axions, gluons, tachyons, etc. and until recently the Higgs boson).

\section{Materials and Methods}

\subsection{Physics of GV Technology}

These super small particles are closely related to scalar particles introduced into the Peccei-Quinn theory [3], which actually condense into vacuum and break the Peccei-Quinn symmetry [4]. These particles are very light and very weakly coupled to other matter; therefore, these super small particles require an interpretation of quantum mechanics to reflect that current standard methodologies do not fully explain quantum conduct in either the theoretical or real world. This is not new itself. Almost all-modern quantum research acknowledges that much more is occurring inside the field than can be explained with old theories. Accepted science often lags the reality of functional science. For example, the compass was accepted as a valuable tool for 300 years before science could accurately describe its mechanism.

Currently, U.S. university researchers refer to the fields created by elementary particles as "subatomic", and identify four main interactions. The strong force; or strong interaction (strong nuclear force, or color force) is one of the four fundamental interactions of nature, others being electromagnetism, the weak interaction and gravitation [5-7]. At the atomic scale, strong interaction is about 100 times stronger than electromagnetism, which is several orders of magnitude stronger than weak force interaction and gravitation.

Although small in size and great in number, the atom is one of the greatest enigmas in the science world today. The most basic parts of the atom are the electron, the proton and the neutron. These three make up a small group of the known subatomic particles. Of these three, only the electron is actually a fundamental particle. The proton and neutron are both hadrons composed of different smaller particles called quarks. Any of the subatomic particles that are formed from quarks, and thus react through strong nuclear force, are hadrons. The hadrons include mesons and baryons, which are quark-based particles.

As quark-based particles, baryons participate in strong interaction, whereas leptons, which are not quark-based, do not participate in strong interaction. All known subatomic particles except bosons and leptons are hadrons. Except for protons and for neutrons that are bound in nuclei, all hadrons have short lives and are produced in the high-energy collisions of subatomic particles. The other three basic 
forces of nature also affect hadron behavior: all are subject to gravitation; charged hadrons obey electromagnetic laws; and some hadrons break up by way of the weak nuclear force, while others decay via the strong electromagnetic forces.

Schools in Russia call these fields created by elementary particles quantum (wave) fields and similarly consider four types of interactions. Data reflected that the fields are analogous in their properties to MLF (microlepton fields) and are called "orgone fields", “torsional fields", "scalar fields", "energy-information space”, etc.. The effects of what A.F. Okhatrin identified are slowly being matched by current research. By applying both new particle theory and refined interpretation of quantum mechanics to the search for signatures of precious metals and hydrocarbons, benefits have been obtained.

\subsection{GV Technology Background}

As regards GV Technology, in addition to the observed effects, two assumptions are fundamental:

(1) All substances which are to a lesser or greater degree in an excited state, and not only radioactive ones, are subject to natural decay. All decay leaves traces of its passing.

(2) The Earth is a strong natural generator of a constant electromagnetic field. All substances, e.g., of the Earth's crust, are already in an excited state. As an analogy, one can give the example of using NITON, DELTA and other portable spectrometers. As a result of the effect of X-radiation, forced excitation of the surface of the investigated sample takes place, followed by detection and dispersion into an "intrinsic radiation" spectrum.

In this way, all elements exist in an excited state and are constantly emitting what could be called their "intrinsic radiation". The resulting clusters and macro-cluster field structures are sometimes observed as a result of polarization above the Earth's surface. These are described in US Patent 4005289, 250-252, 1976, and have been recorded above an oil field in
Russia from an orbiting satellite. As practice has shown, such cluster structures, which apparently have a spherical shape initially, form something of a pear-like shape, apparently as a result of gravitational and other effects. Thus, "prominent" directions of propagation no doubt exist. Furthermore, as practice has shown, clusters vary in structure, which apparently reflects variegation of natural formations.

Cluster and macro-cluster structures of 3SPs most likely exist also inside bodies, participating in processes of their transformation, deformation, destruction, and affecting their characteristics.

\section{Results and Discussion}

\subsection{Comparison of GV Technology to Other Survey Technologies}

Exploration and delineation of lode and placer gold is initiated by obtaining mineral rights for a specific area, followed by the preparation of a good geological map of the area of interest, initially on a regional scale $(1: 50,000)$ by completing a number of field geological traverses, and with the aid of aerial photographs and satellite imagery (e.g., Ref. [2]). Geologists collect rock chip samples from favorable locations for gold mineralization while preparing their geological map. Geochemical methods (e.g., Refs. [2, 8]) will be included in the fieldwork, including stream sediment sampling and soil sampling to define anomalous areas. Similarly, geophysical methods (e.g., Refs. [2, 8]) can be deployed to locate any conductive or magnetic bodies below the soil horizon. Depending on the sampling results, the target areas are delimited for detained sampling in three dimensions. TD (three-dimensional) samplings of gold bearing zones are carried out by diamond core drilling and/or RC (reverse circulation) drilling. These cores and rock chips are submitted for assay and based on the three-dimensional sampling of the gold-bearing zone (ore body), the length, width, depth and grade of the gold deposit is estimated.

In comparisons to these more traditional methods 
used for gold exploration, GV technology has particular and unequalled advantages:

- GV technology is ecologically friendly.

- It is profoundly less expensive than present gold exploration methods.

- It is significantly faster than current technologies. Estimated time to conduct a complete analysis of a given area $100 \mathrm{~km} \times 100 \mathrm{~km}$ is normally 5 months to 6 months instead of 2 years to 3 years.

- The technology is highly accurate in defining and delineating mineral deposits both laterally and vertically.

GV technology has three registered patents and has received several national and international awards. It is a superior technology because GV technology counters the inherent uncertainty in exploration and the high cost of failure. The cost of every successful exploration technology and well drilling success bears the burden of all the failures that precede it. The cost of finding a productive ore body becomes increased when burdened with low success rates in identifying gold deposits with standard exploration types of exploration. In addition, exploration drilling is expensive because it depends on the environment, depth, and complexity of the mineral resource.

However, GV technology identifies, with high probability, the exact location of gold deposits. Thus, the technology provides the opportunity to:

- Control cost and time of discovery for extensive gold lease positions;

- Conduct an in-depth evaluation from satellite imagery to near surface surveys;

- Obtain critical data about mass and depth of deposits, prior to extensive exploration;

- Conduct a full feasibility study, without the need for any indirect or direct techniques such as drilling.

As a result, this technology achieves multiple reductions in cost. Exploration expenditures are more efficient and can lead to the end of all "dry hole drilling”. GV technology is ecologically friendly, thus providing a considerable reduction in environmental mitigation costs.

\subsection{GV Technology Overview}

GV technology is applied in three separate phases: Phase 1-initial identification of anomalous deposits for a defined area based on data obtained from satellite imagery; Phase 2-a field expedition (usually a helicopter survey of the area) and data processing; and Phase 3-final report on lode deposit and drilling recommendations for gold-bearing zones.

\subsubsection{Phase 1-Deposit Identification}

When the earth's surface is photographed from space (Fig. 1), halides of the emulsion layer of the satellite imagery record not only an image of the terrain in the visible optical spectrum, but also the effect of energy clusters formed by natural objects.

The physical/informational foundation for prospecting underground resources is based on the theory of energy-information exchange in geosystems. This reflects the known properties of substances to exhibit dipole fields when placed in electric fields.

The first phase of GV technology consists of assembling the satellite images (Fig. 2) for laboratory examination (Fig 3) and is based on the well-known

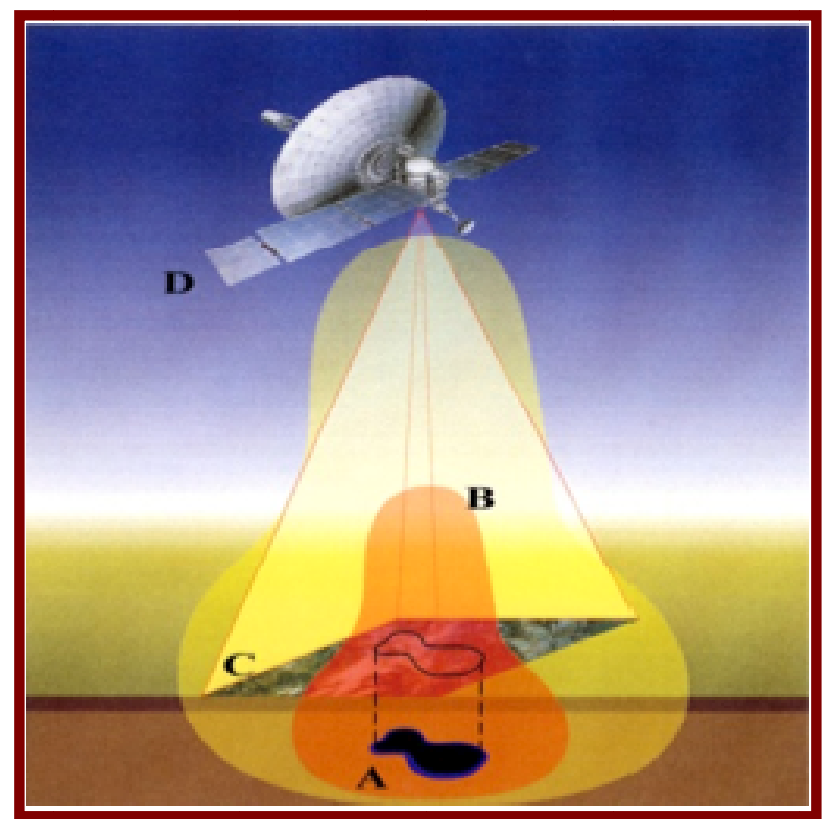

Fig. 1 Macrocluster structure are recorded from a satellite traveling in orbit. 
effect of a photochemical reaction in halide microcrystals, which occurs under the influence of electromagnetic waves (of the visible, infrared, and ultraviolet spectrum), as well as the effect of charged particles (recording of rare events). The conclusion is that halide reacts both to particles and to waves. A natural formation constitutes a geophysical waveguide, consisting of emitters of different magnitude that are excited by a broad spectrum of active frequencies. Thus, each emitted wave of naturally induced polarization has its own amplitude, frequency and phase.

As a result of their interaction, each natural formation will form, on the observation surface, an interference image of its total field of natural polarization, with a spatiotemporal structure characteristic of the object (Figs. 1 \& 3). In this way, the spatiotemporal structure of a wave field of naturally induced polarization of energetically active natural formations presents an image of the material/energy structure of natural objects on the earth's surface.

Visualization of natural formations being searched for (Phase 1 of GV technology) is achieved as a result of the resonant expression of information carried by the emulsion layer of the satellite image, and an optical-subatomic filter tuned to a specific chemical element or compound thereof (gold, petroleum, gas, silver, etc.). Despite the similarity of chemical composition, the "intrinsic radiation" spectrum has several differences, which make it possible to distinguish the carbon of natural coal from the carbon of diamonds, etc.. The first phase of GV technology will determine whether the target underground resource is present in the surveyed territory and how it is territorially confined as represented by the yellow objects, indicated in Fig. 4.

In other words, in the first phase for the application of the GV Technology the collected information is processed using a special software program to identify the location of anomalous deposits (Fig. 3). Data will be analyzed and narrow survey areas, which include the anomalous prospective parcels of land, will be recommended for the second survey phase including the helicopter survey. However, the results of Phase 1 allow one only to establish the fact of whether the target underground resource (i.e., gold) is present in

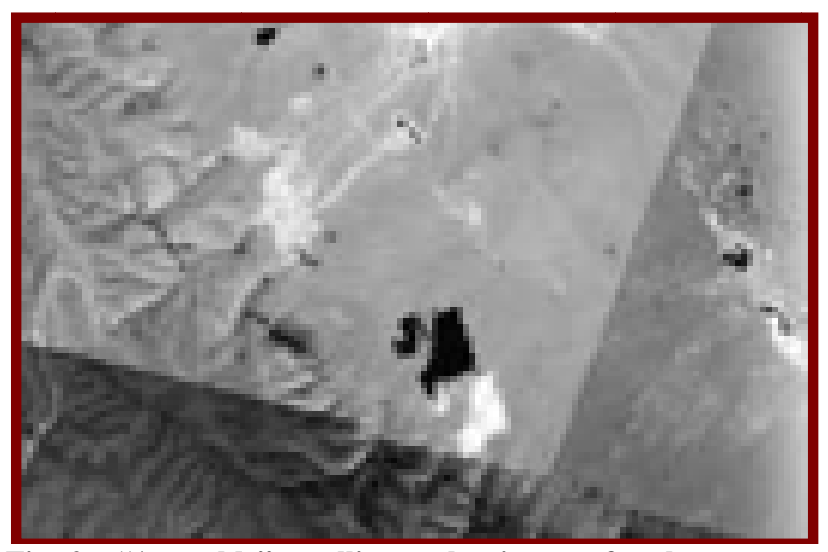

Fig. 2 “Assemble" satellite analog images for the area to be evaluated.

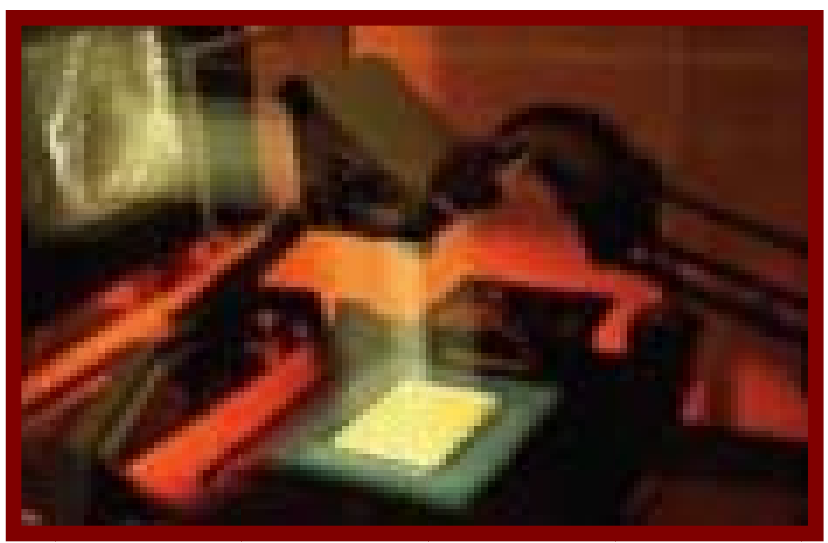

Fig. 3 Laboratory method to identify individual emanations from gold deposits.

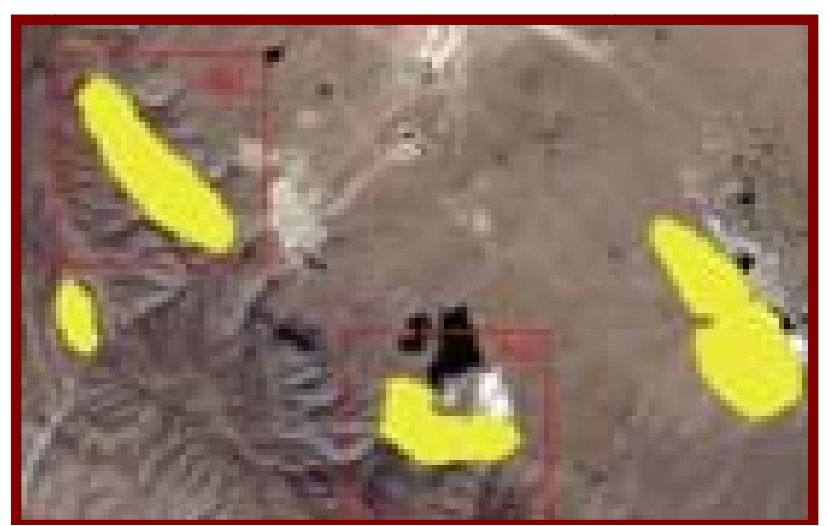

Fig. 4 Gold anomalies are identified from the digital satellite film processing. 
the surveyed territory and how it is territorially confined.

3.2.2 Phase 2-Field Expedition and Data Processing

In the second stage of GV Technology applications, a field survey consists of flying a helicopter (Fig. 5) over the identified resource (i.e., gold) anomalies to provide customers with information of the exact location of deposit outlines and bedding depth of productive horizons. The field expedition includes measuring anomalous deposits using the GVI proprietary GEO-SCAN ML2 detector, installed onboard a helicopter or, in some cases, if the areal extent of survey is quite limited by ground transport.

Phase 2 processing creates digital imaging of the fields, their intensity, and areal extent (Fig. 6), to provide data for conducting mathematical and cartographic modeling of the deposits and to identity the anomalous areas for the construction of a spatial model of the deposit and preliminary estimation of reserves during the completion of Phase 3.

3.2.3 Phase 3-Final Reporting and Drilling Recommendation

In Phase 3, with additional data processing, different depths of gold deposits are defined, using the superposition principle (Fig. 7).

Additionally in Phase 3, field expedition survey results and coordinates of the gold anomalies are presented on maps identifying varying depths and amounts of gold in ounces per ton with recommendations of core drilling locations. The combined result is a tightly focused map that shows where all the emitter masses are residing and how deep they exist (Fig. 7). The complex analysis of information received by GV technology surveys in combination with other geological and geophysical methods allows customers increasing precision in forecasting and identifying mineral deposits.

The scale of the intensity of intrinsic emission using GV technology is usually indicated in four or so colors (Fig. 8), which correspond to the gradational characteristic for the specific anomalous areas being investigated.

High concentration points recommended for drilling gold bearing deposits are designated by yellow colors

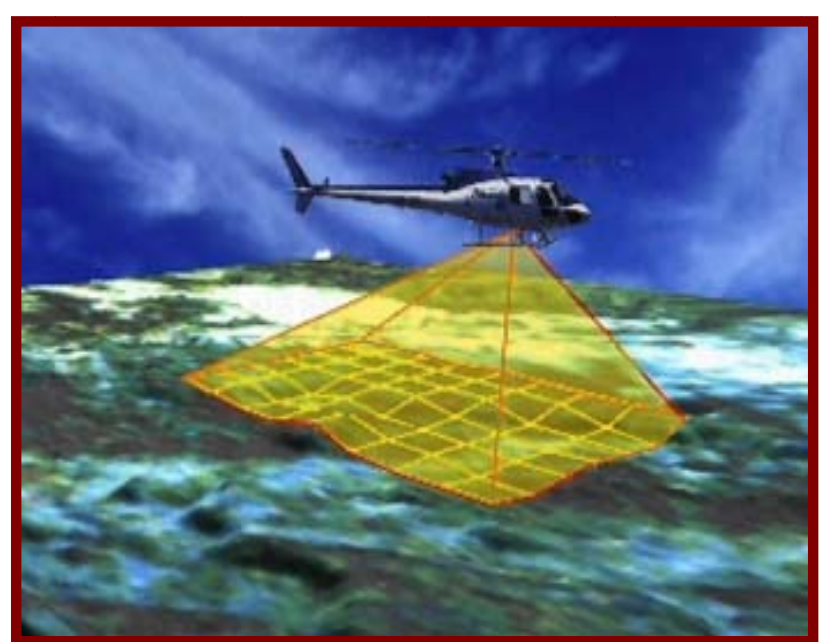

Fig. 5 Field survey consists of flying helicopter over identified gold anomalies.

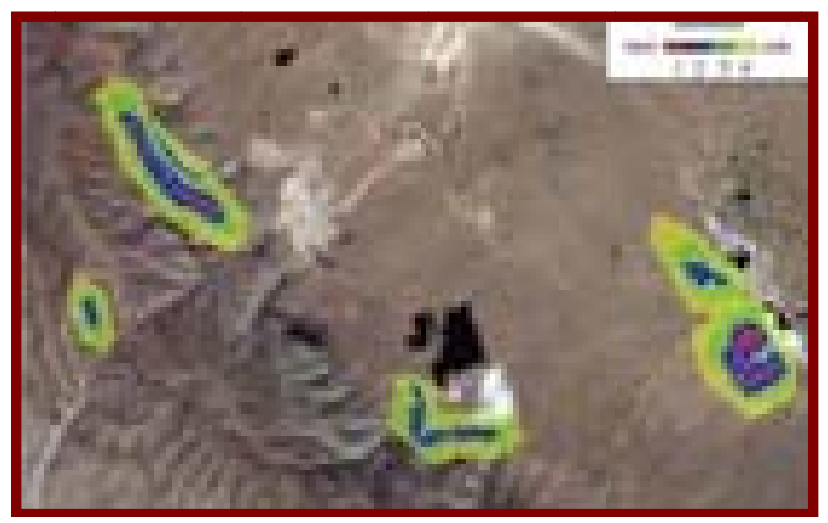

Fig. 6 Maps of field expedition survey result and coordinates of gold anomalies.

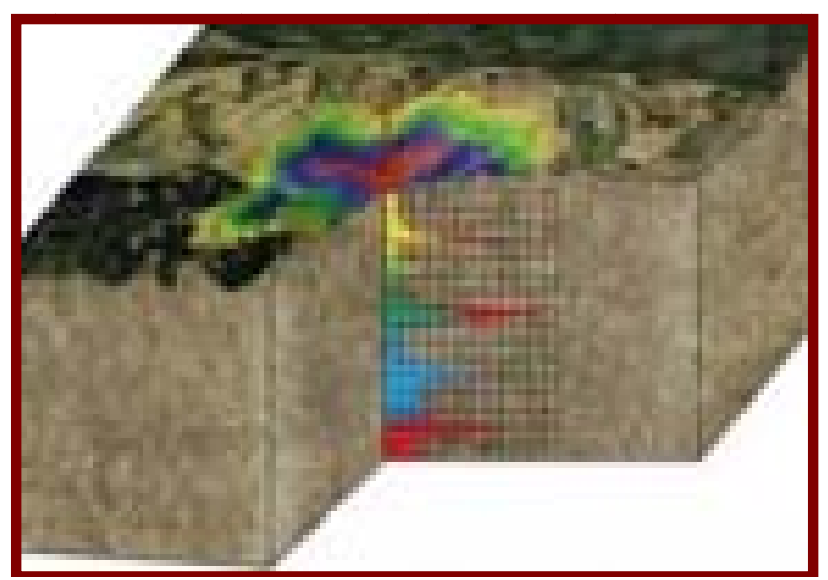

Fig. 7 Varying depths of gold deposits with recommended drilling locations. 


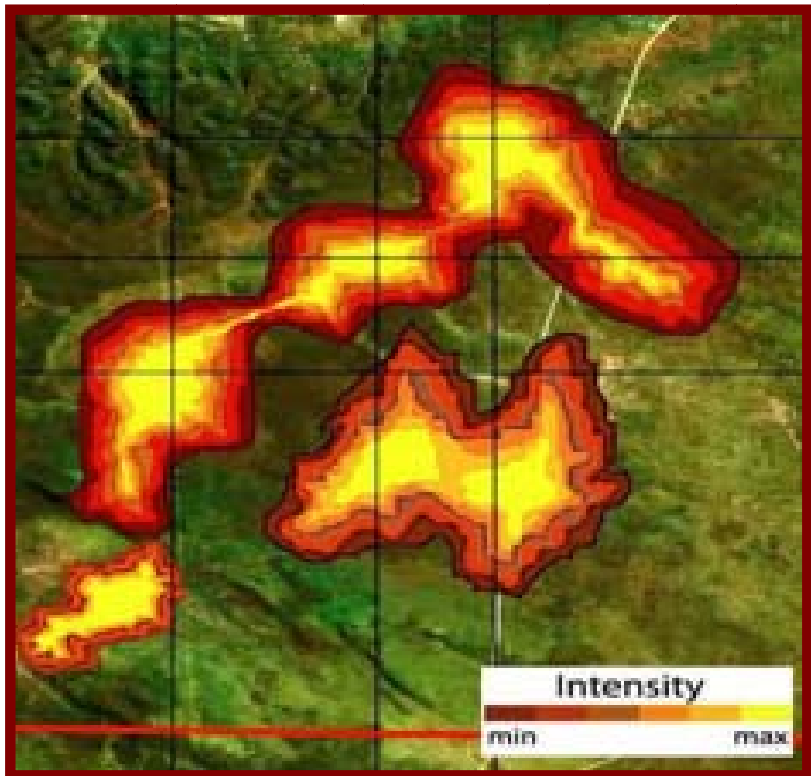

Fig. 8 Drilling recommended at the identified GV Technology gold anomalies.

as indicated in Fig. 8. Orange colors also represent recommended areas for drilling by the customer's drilling specialists and geologists.

\subsection{GV Technology Gold Deposit Case Histories}

During the last nineteen years, GVI has successfully completed several GV technology survey contracts for gold bearing deposit identifications on five continents. With permission from customers, abbreviated case histories of several international surveys using GV technology for gold prospecting are presented in this section.

\subsubsection{5-2007}

Kirghizia at the At-Bashi District was surveyed for ZAO International Mining Company with the intent to define gold deposit locations. The customer used survey results completed by conducting a Phase 1 survey over the area indicated in Fig. 9. and discovered 15 gold sites with high gold concentrations.

After analyses of data obtained from Phase 1 (Fig. 10) of GV Technology survey, customer drilled several diamond core test holes. After test, drilling and core assay analyses reserve estimation identified a deposit of 11 tonnes of gold as a proven reserve with $5 \mathrm{~g} / \mathrm{t}$ to $9 \mathrm{~g} / \mathrm{t}$, which was sold in one year to another mining company.

\subsubsection{5-2011}

Mongolia for Russian Company OOO IEE Zarubezhcvetmet. A photograph of GV Technology survey area is presented in Fig. 11.

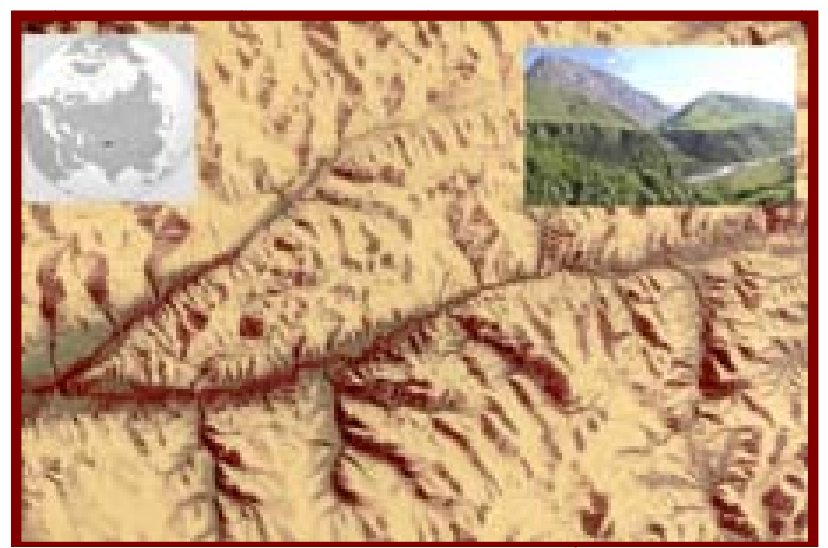

Fig. 9 GV technology surveyed $300 \mathrm{~km}^{2}$ in the At-Bashi area of the Republic of Kirghizia.

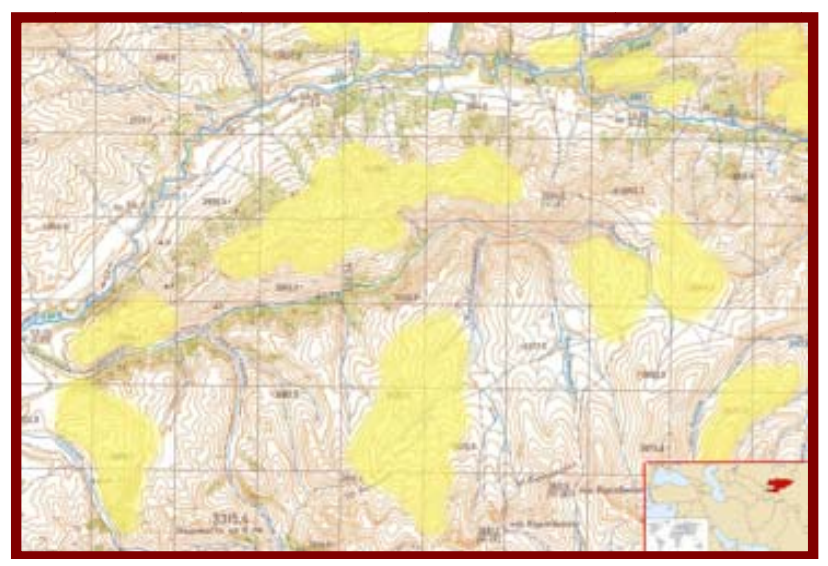

Fig. 10 Gold anomalies identified in Phase 1 of GV technology survey in Kirghizia.

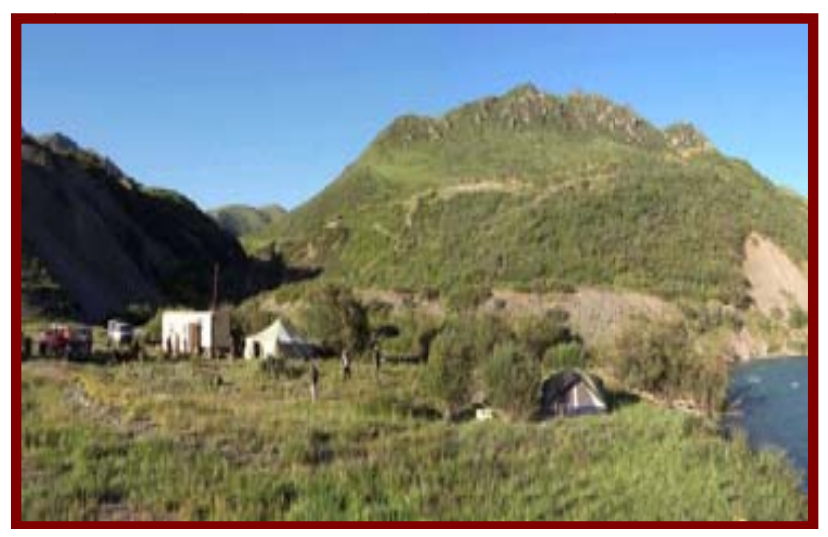

Fig. 11 Photo of survey area in Mongolia. 
A GVI crew conducted all three stages of GV technology on $300 \mathrm{~km}^{2}$ to identify gold deposits. After analyses of data obtained from both Phases 1 and 2 (Fig. 12) of GV Technology survey, GVI estimated that the customer had a gold deposit of at least 19 tonnes.

After drilling several diamond core test holes (Fig. 13), reserve estimation by the customer identified more than 19 tonnes of gold as a proven reserve. In fact, gold was discovered on all sites identified by the GV technology surveys.

Based on results and analyses of the first GVI survey, a Mongolian company, Sansarin Geological Haigool, signed a 5 year Survey Contract with GVI for further GV technology surveys. During the five years GVI conducted 17 successful surveys for gold, copper, hydrocarbons, and water in Mongolia.

GVI also conducted surveys for copper deposits on $350 \mathrm{~km}^{2}$ for one of the largest companies, East Asia Copper Mining Company Erdenet in additional areas of the Mongolia region. The first GV technology survey resulted in the identification and delineation of rich copper bearing deposits at 4 areas.

The most successful GV technology survey for identification of gold deposits in Mongolia conducted in the Bayanhogor Province. Satellite image of Bayanhogor gold prospect is presented in Fig. 14.

With the analysis of the results from Phase 1 of the GV technology survey, there was a decision made to continue with a Phase 2 helicopter survey. A photograph of the crew and helicopter used in the Phase 2 survey is presented in Fig. 15.

A map of results and analyses of the Phase 2 field expedition survey at Bayanhogor is provided in Fig. 16. After internal analyses of data obtained from the GV technology survey, several diamond core test holes were drilled to confirm the identification of the gold bearing deposit and mining was immediately started.

A satellite image of the mining site is shown in Fig. 17, with a photograph of the mining site presented in Fig. 18. In summary, the mine had produced 2,000 tonnes of gold by the end of 2009 .

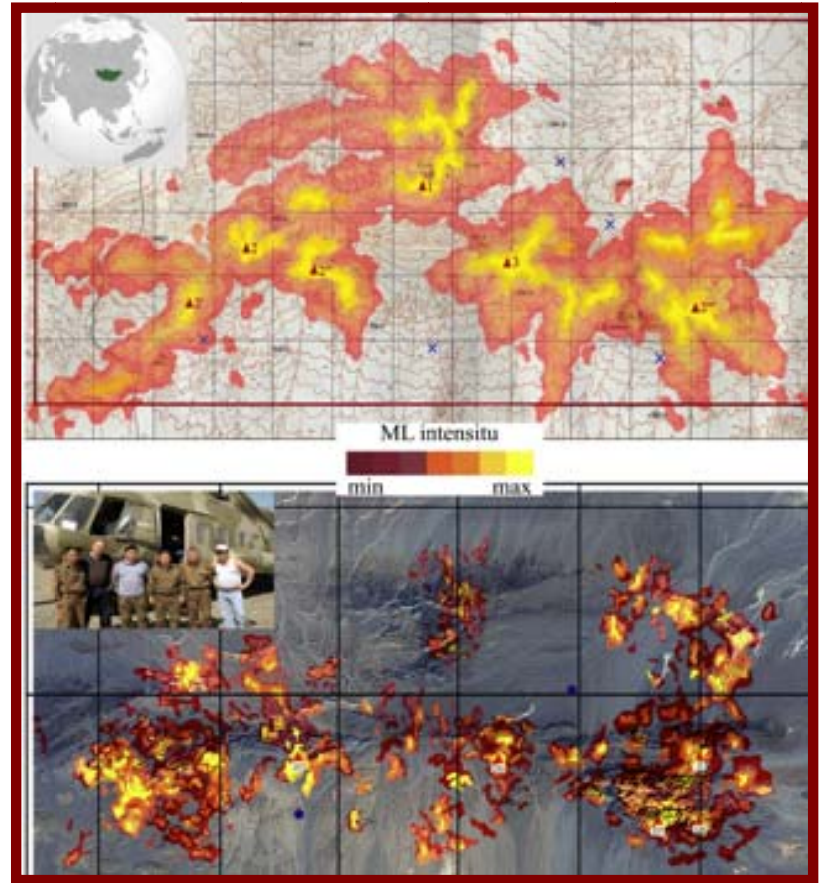

Fig. 12 Results from Phase 1 and Phase 2 GV technology gold surveys in Mongolia.

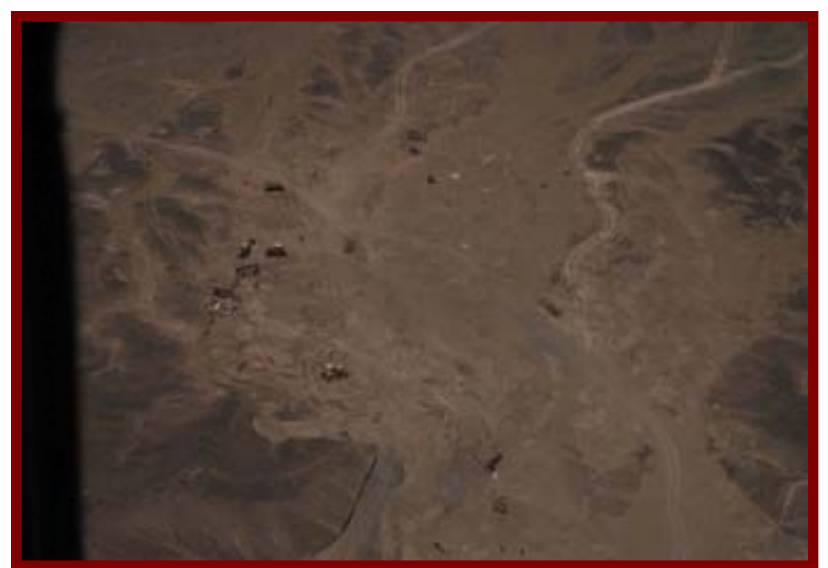

Fig. 13 Satellite image of diamond core test drilling by Zarubezhevetmet based on GV Technology survey results.

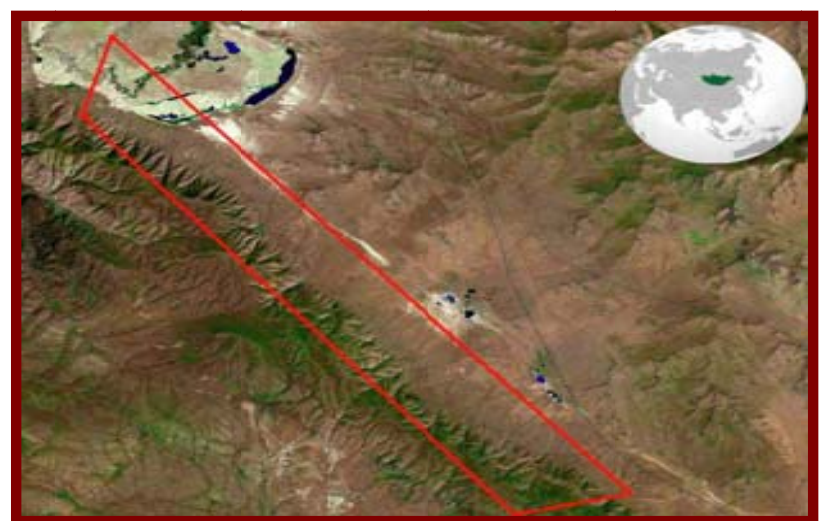

Fig. 14 Satellite image of the Bayanhogor gold prospect in Mongolia. 


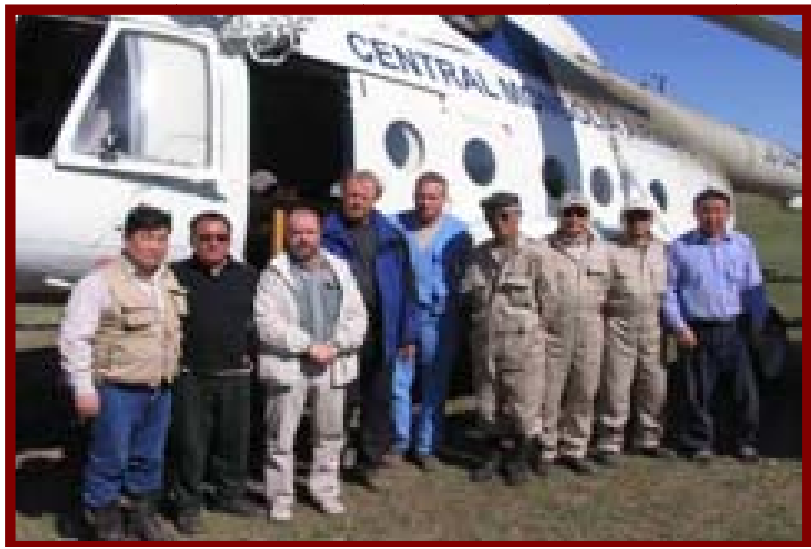

Fig. 15 The Crew and Helicopter used for the GV Technology Phase 2 Survey at Bayanhogor.

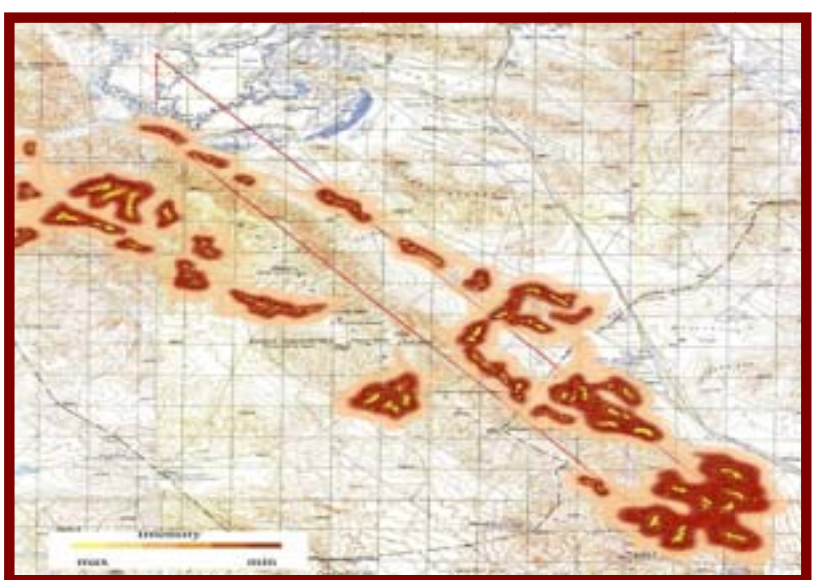

Fig. 16 Results from Phase 2 GV technology survey-helicopter survey and analyses of potential gold bearing deposits at Bayanhogor.

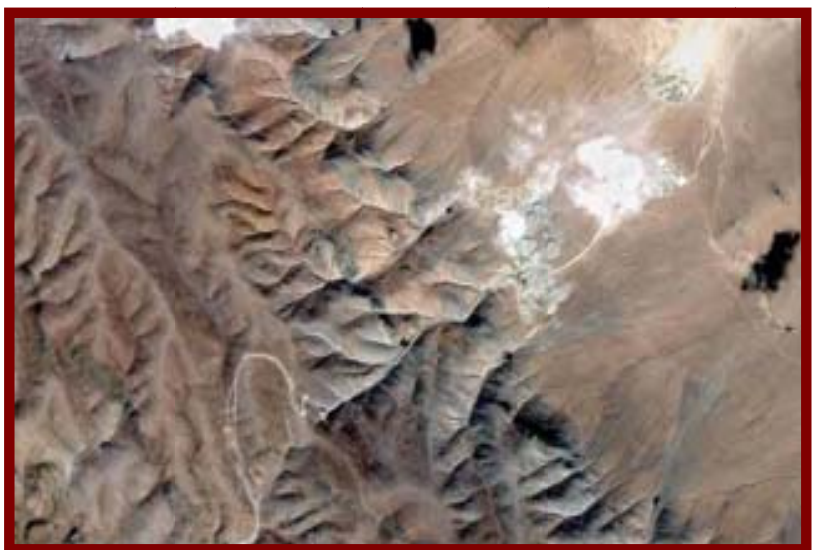

Fig. 17 Satellite image of the gold mining site at Bayanhogor.

\subsubsection{6}

Namibia, a country located in southern Africa, and the customer was Namslavic Exploration. After analyses of data obtained from Phase 1 (Fig. 19), GVI identified 11 prospective sites for future gold exploration and 4 sites for diamonds. Namslavic requested Phase 2 (Fig. 20) surveys at 2 of the most prospective sites in the Chirimbo area where high concentrations of gold were identified. Customer continued exploration, by applying geochemical and electromagnetic methods as well as shallow core drilling to delineate large gold deposits.

\subsubsection{6-2012}

Tanzania, for Russian-Tanzanian company, Geo Nadir, completed gold surveys in the southeast of Dar es Salaam area. The Phase 1 survey was conducted on an area of about $1,000 \mathrm{~km}^{2}$. Prospective gold deposits were determined in $350 \mathrm{~km}^{2}$ and the reduced area was recommended for a Phase 2 GV Technology survey (Fig. 21).

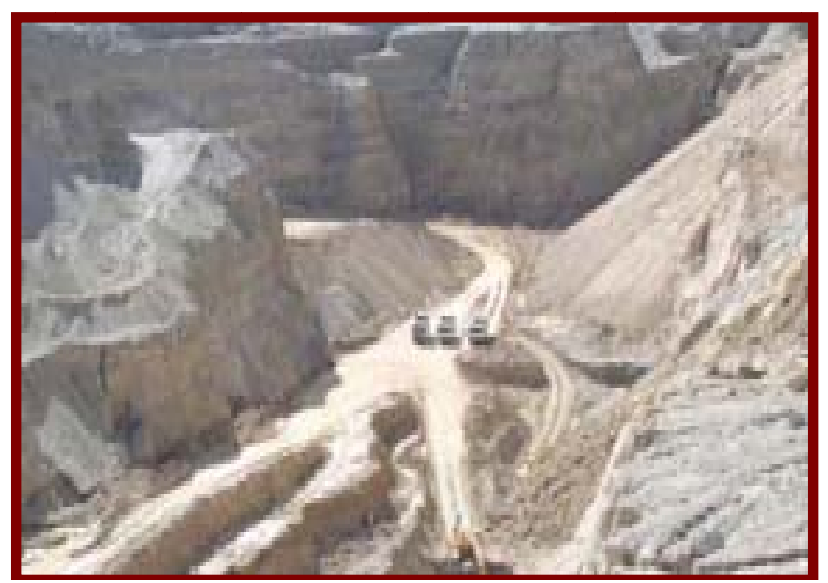

Fig. 18 Photograph of the gold mining site at Bayanhogor.

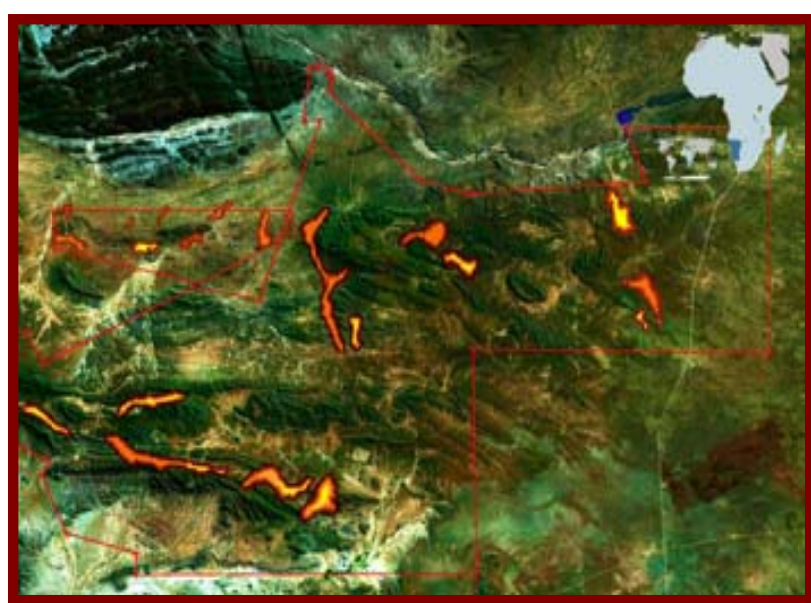

Fig. 19 Results from Phase 1 GV technology survey-satellite image analyses in the Okavago area of Namibia. 


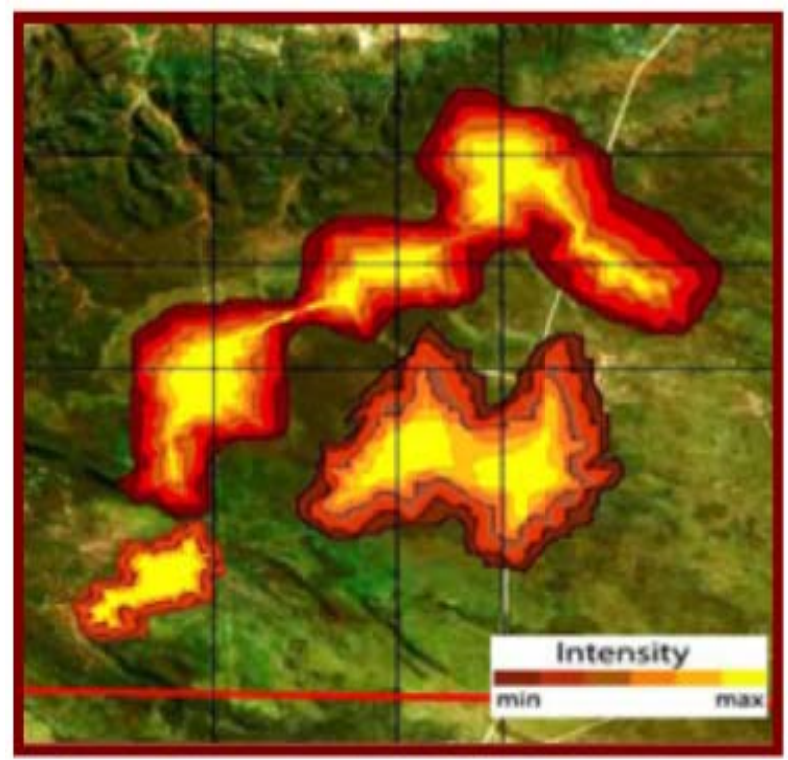

Fig. 20 Results from Phase 2 GV technology survey-helicopter survey and analyses.

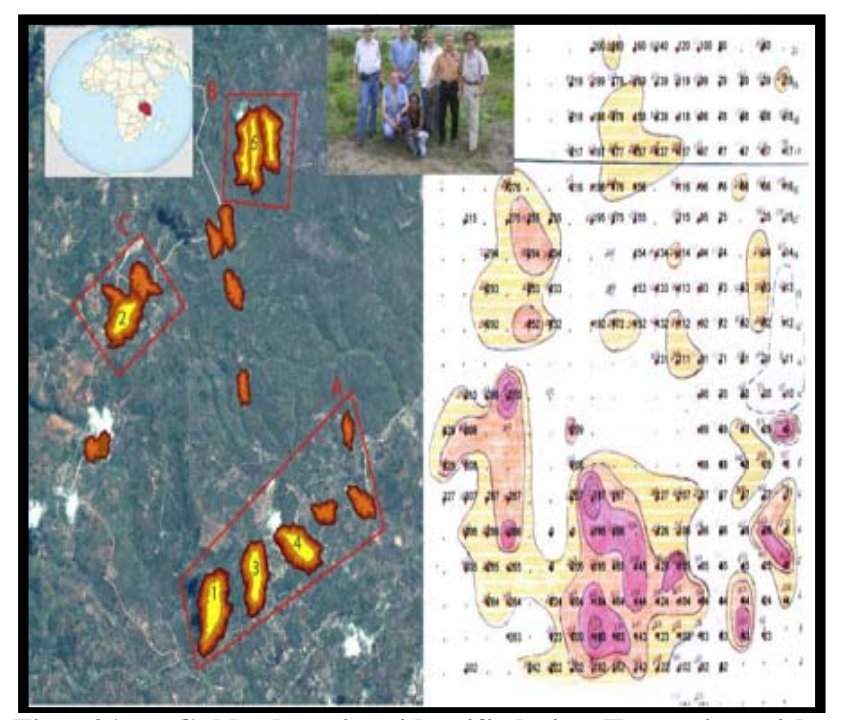

Fig. 21 Gold deposits identified in Tanzania with three-phase GV technology surveys.

Further, geochemical and geophysical surveys of the area as well as additional mapping proved that the GV Technology survey gold discoveries were 90+\% correct compared to the additional data that was obtained by the Customer. Gold concentrations in areas recommend by GVI for core hole drilling and assay analysis confirmed observations of $1.5 \mathrm{~g} / \mathrm{t}$ to $32 \mathrm{~g} / \mathrm{t}$. In 2012, the gold deposit defined by GV technology was classified as large. GVI surveyed and registered claims for 3 sites with gold discovered on all three and sold the claims to a Chinese mining company for further development.

\subsubsection{9-2013}

United States in Lander County, Nevada. GVI conducted all three phases of GV technology surveying for DDSLLC (direct detection services LLC). Although there are more than 700 gold mines located in Lander County, there remain many square miles of unexplored federal lands. In 2010, DDSLLC decided to utilize GV technology to define gold lode claims. DDSLLC has identified, delineated, staked, and acquired 27 gold lode claims on federal lands administered by the U.S. BLM (bureau of land management). At least, 25 gold anomalies were identified as the results of the analysis of Phases 1 and 2 of the GV technology survey (Figs. 22a and 22b).

Based on the detailed analysis of Phase 3 of the GV technology survey (Fig. 23), the estimate of the productive gold levels start at a depth of 10 meters to 15 meters and extend to a depth of 210 meters to 240 meters, with an estimated 7 productive horizons to 10 productive horizons. Initial estimate of gold content is from 3 grams to 9 grams per ton of ore with an estimate of 17 tons to 17.5 tons of gold.

Gold concentrations estimated by the GV Technology survey have not been confirmed by additional core drilling (Fig. 24) and assay analyses for all GV technology gold anomalies.

An exploration program is planned to effectively test the subsurface gold zones. This program will consist of as many as thirty core holes and assay test work. DDSLLC plans to convert these initially identified gold lode claims to mineable projects which will then be sold to gold mine development company.

\subsubsection{3}

United States in Mineral County, Nevada. GVI conducted a Phase 1 survey on the Fletcher Junction gold prospect of Nevada Exploration Inc. Ref. [9], which was obtained from the NGE web site. In order to complete a Phase 1 evaluation of the Fletcher Junction prospect, an area of approximately $15 \mathrm{~km}$ by $15 \mathrm{~km}$, or about 225 square kilometers, was selected with the NGE leases essentially in the center 


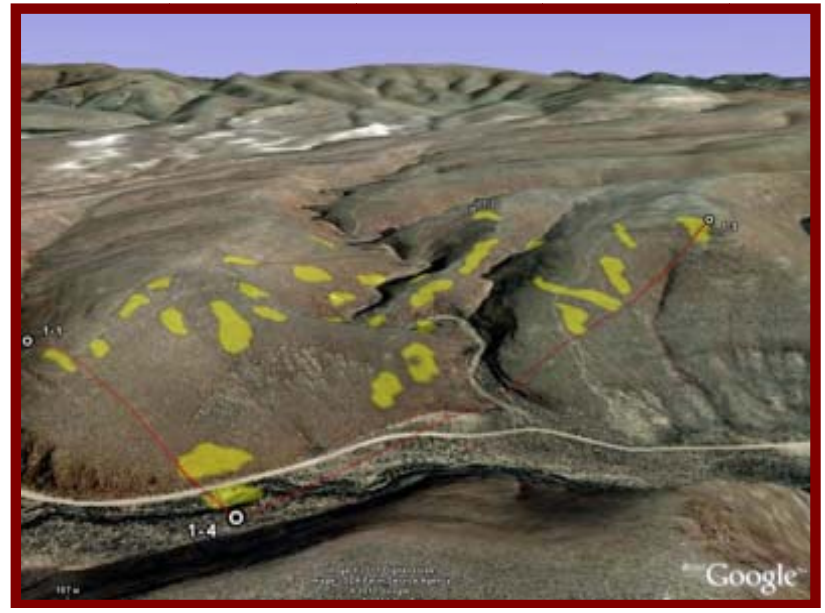

(a)

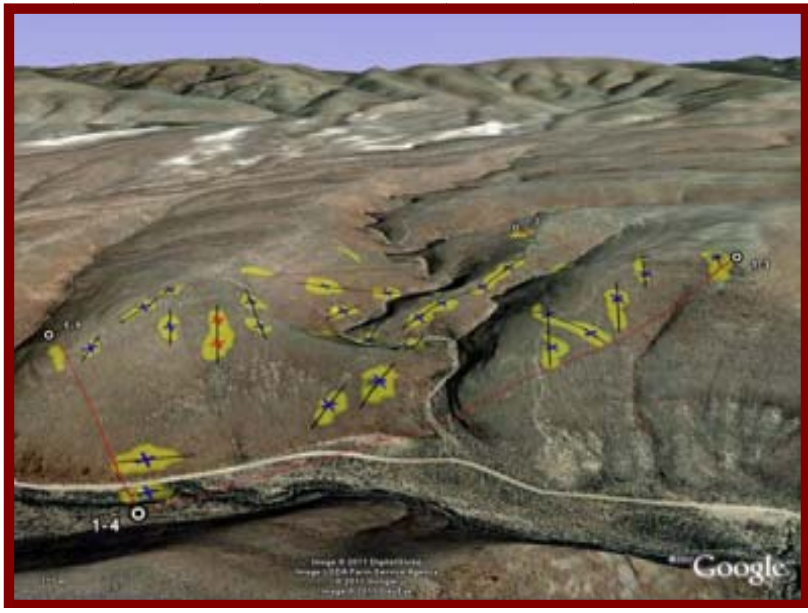

(b)

Fig. 22 (a) Gold anomalies identified by Phase 1 GV technology survey in Lander County, NV; (b) Gold anomalies from Phase 2 survey at Lander County, NV with red crosses in left center indicating first core drilling locations.

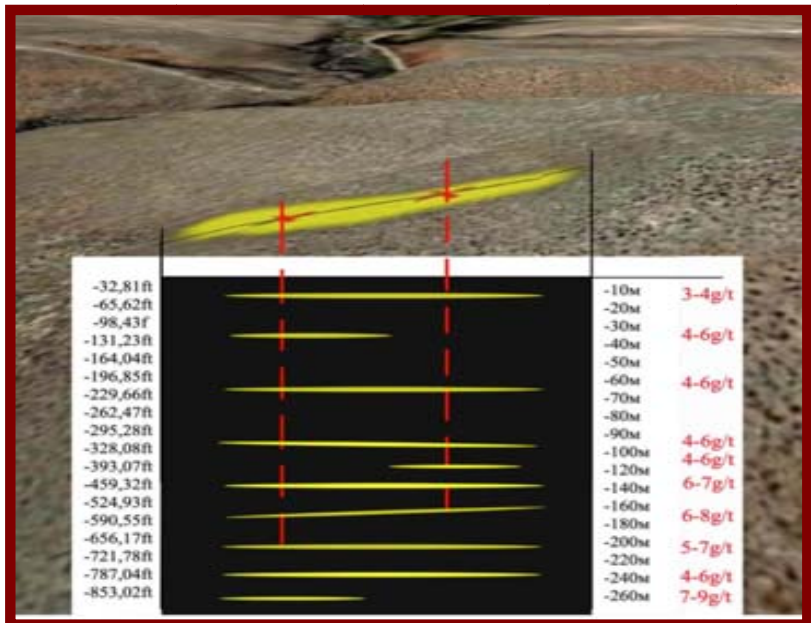

Fig. 23 First core holes had 10 levels of gold and predicted gold content ranged from $3 \mathrm{~g} / \mathrm{t}$ to $9 \mathrm{~g} / \mathrm{t}$.

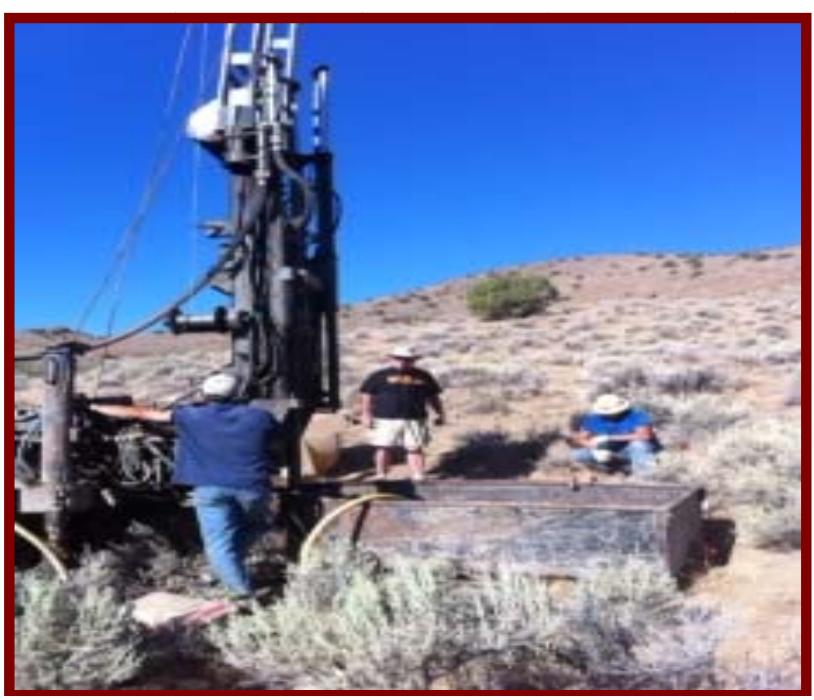

Fig. 24 Drilling at Lander County prospect.

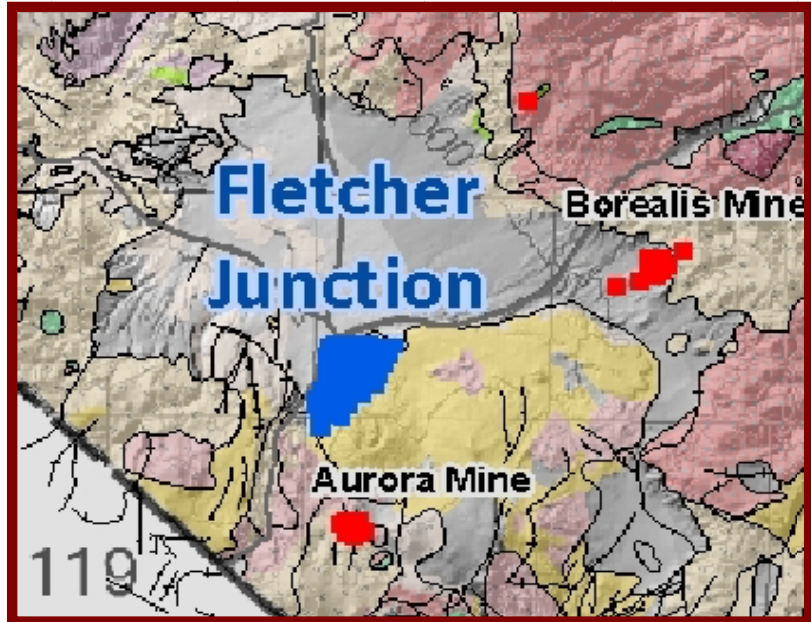

Fig. 25 Fletcher Junction gold leases of NGE in blue and mines are designated in red.

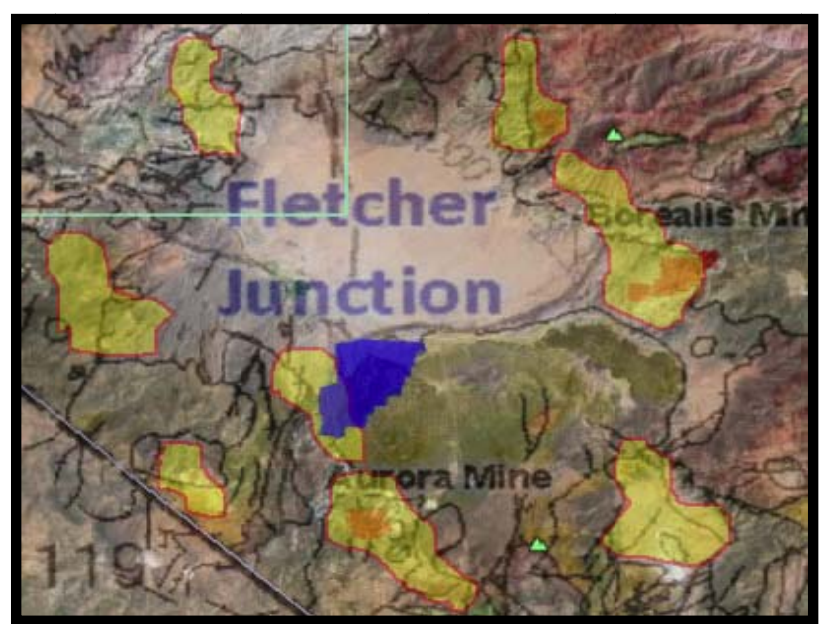

Fig. 26 Phase $1 \mathrm{GV}$ technology survey evaluation of the Fletcher Junction gold leases and immediate mines with eight anomalous gold areas identified. 
of the survey area (Fig. 25).

Based on analysis of satellite imagery of the area, GVI determined there are 8 anomalous gold areas (Fig. 26), which included an anomaly associated with the NGE leases and anomalies over the Aurora Mine, the Borealis Mine, and a small mine in the upper right side of Figs. 25 and 26. GVI recommends that NGE, or another gold developer, follow the Phase 1 data with Phase 2 and $3 \mathrm{GV}$ technology surveys to provide better definition of the gold zones and drilling locations in several of the other anomalous gold areas identified.

\section{Conclusions}

Based on the lode gold deposit case histories, we have received permission to publish, the use of GV technology has been very successful in identifying and delineating lode gold deposits both laterally and vertically. GV technology is a superior technology because it counters the inherent uncertainty in exploration and the high cost of failure. The cost of every successful exploration technology and well drilling success bears the burden of all the failures that occurred previously. The cost of finding a productive ore body becomes increased when burdened with low success rates in identifying gold deposits with standard types of exploration. In addition, exploration drilling is expensive because it depends on the environment, depth, and complexity of the mineral resource.

GVI recommends the application of our technology to the exploration for and delineation of gold deposits being sought by mining companies. GVI is convinced that once companies have used our GV technology, it will become an accepted exploration and delineation tool and will provide excellent data to better define the lateral and vertical extent of the gold deposits and provide successful core drilling locations that are being sought. GVI continues to anticipate that companies that determine to utilize our GV Technology will improve their success ratio associated with the exploration for and delineation of the lateral and vertical extent of gold deposits as well as better define successful drilling locations. We can further assure mining companies that the cost associated with the definition of lode gold deposits will be decreased with the use of GV technology and since GV technology is ecologically friendly, it provides a considerable reduction in environmental mitigation costs.

\section{References}

[1] Strategies for Gold Reserve Replacement: The Cost of Finding Gold, Metals Economics Group, 2006.

[2] F. Robert, R. Brommecker, B.T. Bourne, P.J. Dobak, C.J. McEwan, R.R. Rowe, et al., Models and exploration methods for major gold deposit types, in: Proceedings of the Exploration 07: Fifth Decennial International Conference on Mineral Exploration, Canada, 2007, pp. 691-711.

[3] R.D. Peccei, H.R. Quinn, CP conservation in the presence of pseudoparticles, Physical Review Letters 38 (25) 1440.

[4] R.D. Peccei, H.R. Quinn, Constraints imposed by CP conservation in the presence of pseudoparticles, Physical Review D 16 (6) (1977) 1791.

[5] K.S. Krane, Introductory Nuclear Physics, 3rd ed., Wiley, 1987, p. 864.

[6] K.S. Krane, Modern Physics, 3rd ed., Wiley, 1996, p. 560.

[7] M. Capaldi, Subatomic World, Standard Model \& Particle Physics [Online], https://pinterest.com/mcapaldi936/subatomic-world-stand ard-model-particle-physics/ (accessed 2013).

[8] R.H. Sillitoe, J.F.H. Thompson, Changes in mineral exploration practice: consequences for discovery, Society of Economic Geologists special Publication (12) (2006) 193-219.

[9] W. Hodges, Fletcher Junction project regional geologic context, 2013, Nevada Exploration Inc. Web site, http://www.nevadaexploration.com/properties/fletcher/ge ology/ (accessed 2013). 